\title{
Buckling Analysis of Non-Local Timoshenko Beams by Using Fourier Series
}

\author{
Hayrullah Gün Kadıoğlu ${ }^{a^{*}}$, Mustafa Özgür Yaylı ${ }^{b}$ \\ ${ }^{a, b}$ Uludăg University, Faculty of Engineering, Department of Civil Engineering, Bursa, Turkey. \\ *E-mail address: hyrllh_1113@ hotmail.com \\ ORCID numbers of authors: \\ 0000-0001-7370-2722a , 0000-0003-2231-170X ${ }^{b}$
}

Received date: December 2017

Accepted date: December 2017

\begin{abstract}
In this study, buckling analysis of a nano sized beam has been performed by using Timoshenko beam theory and Eringen's nonlocal elasticity theory. Timoshenko beam theory takes into account not only bending moment but also shear force. Therefore, it gives more accurate outcomes than Euler Bernoulli beam theory. Moreover, Eringen's nonlocal elasticity theory takes into account the small scale effect. Thus, these two theories are utilized in this study. The vertical displacement function is chosen as a Fourier sine series. Similarly, the rotation function is chosen as a Fourier cosine series. These functions are enforced by Stokes' transformation, and higher order derivatives of them are obtained. These derivatives are written in the governing equations for the buckling of nonlocal Timoshenko beams. Hence Fourier coefficients are acquired. Subsequently boundary condition of established beam model is identified with Timoshenko beam and Eringen's nonlocal elasticity theories, and the linear equations are obtained. A coefficients matrix is created by utilizing these linear systems of equations. When determinant of this coefficient matrix is calculated, the critical buckling loads are acquired. Finally, achieved outcomes are compared with other studies in the literature. Calculated results are also presented in a series of figures and tables
\end{abstract}

Keywords: Timoshenko beam theory, Eringen's nonlocal elasticity theory, elastic buckling analysis, Stokes' transformation, Fourier series.

\section{Introduction}

Nano derives from a Greek word meaning dwarf. A nanometer is a unit of length equal to one billionth of a meter. Nanotechnology being with manipulation of substance on an atomic, molecular, and supramolecular scale is a new science. This new science aims to create a lot of new materials which occurs from many different atoms combined. Therefore the emphasis of nanotech. is increasing step by step. A lot of researches being associated with nanotech. are made in the literature. These researches are made by three methods. These are synthesis, simulation and theoretical. Cost of synthesis and simulation are utmost large. Hence theoretical method is utilized commonly. In this study, buckling analysis of nano sized beams has examined by theoretically. Thus Timoshenko beam and Eringen's nonlocal elasticity theories are utilized together. Timoshenko beam theory is more advanced a version of Euler Bernoulli beam theory. In the Euler Bernoulli beam theory, influence of shear on bending deformation is considered negligible compared to bending moment. Therefore plane sections remain plane and normal to the longitudinal axis during bending deformation. However, in the reality, influence of shear on bending deformation cannot be neglected. Thus Timoshenko beam theory regarding not solely moment but also shear effect gives more realistic 
outcomes than Euler Bernoulli beam theory. Moreover Eringen's nonlocal elasticity theory takes into account the small scale effect.

Once the literature is investigated closely, it is observed that. There are a lot of studies being associated with nonlocal elasticity theory. Studies regarding nonlocal elasticity theory found in the literature are compiled and presented in this part. In order to explain the small scale effect, the nonlocal continuum mechanics has been utilized in [1-3]. Moreover Atomistic simulations and experimental findings have proved a significant small scale effect in the mechanic performance of material at micro and nano scale [4-6]. The long term repercussions of these studies on engineering and science have been felt. Thus many investigators have implemented nonlocal elasticity theory for their studies being associated with bending, buckling and vibration [7-36].

In this study, on the basis of the higher order elasticity theory known as nonlocal elasticity theory, an analytical method is presented for the buckling analysis of nano sized Timoshenko beams with rotational restraints. The vertical displacement function is sought as a Fourier cosine series and the slope of the beam is represented as Fourier sine series. Then Stokes' transformation is used to take care of the rotational restraints. A coefficient matrix including shear correction factor and rotational spring parameters is obtained. The eigen values of this matrix gives the buckling loads. Present results are compared with the similar problem solutions available in the literature.

\section{Formulation of the Problem}

According to the nonlocal Timoshenko beam theory, governing equations are given [7];

$$
\begin{gathered}
E I \frac{\partial^{2} \theta}{\partial z^{2}}+\gamma^{2} P \frac{\partial^{3} \varphi}{\partial z^{3}}-\kappa_{S} G A\left(\theta+\frac{\partial \varphi}{\partial z}\right)=0 \\
\kappa_{S} G A\left(\frac{\partial \theta}{\partial z}+\frac{\partial^{2} \varphi}{\partial z^{2}}\right)-P \frac{\partial^{2} \varphi}{\partial z^{2}}=0
\end{gathered}
$$

where, $\mathrm{E}$ is modulus of elasticity, $\mathrm{I}$ is moment of inertia of beam, $\mathrm{G}$ is modulus of elasticity in shear, A is cross sectional area, $\mathrm{b}_{\mathrm{s}}$ is Timoshenko shear coefficient, $\mathrm{P}$ is critical buckling load, $\gamma$ is smallscale effect coefficient $\varphi$ is the vertical displacement function, $\theta$ is the rotation function.

In Ref. [7] and [8], the solution of above equations has been presented for rigid boundary conditions. On the other hand, difference of this study from the other studies is that. This study allows to make solution for non-rigid boundary conditions. Therefore the long term repercussions of this study on engineering and science will be felt.

\subsection{Fourier Series}

The displacement and rotation functions can be indicated as following Fourier series.

$$
\begin{array}{ll}
\varphi=\sum_{n=1}^{\infty} A_{n} \sin \left(\alpha_{n} z\right) & 0<z<L \\
\theta=\sum_{n=1}^{\infty} B_{n} \cos \left(\alpha_{n} z\right) & 0<z<L
\end{array}
$$


Where

$$
\alpha_{n}=\frac{n \pi}{L}
$$

In which $L$ is length of beam, $A_{n}$ and $B_{n}$ is Fourier coefficients.

\subsection{Stokes' Transformation}

In order to include non-rigid boundary conditions in the solution of problem a mathematical transform is necessary. Therefore, in this study, Fourier series and Stokes ' transformations are utilized together and included to the solution of the problem with the deformable boundary conditions.

$$
A_{n}=\frac{2}{L} \int_{0}^{L} \varphi(z) \sin \left(\alpha_{n} z\right)
$$

The first derivative of the displacement function yields;

$$
\varphi^{\prime}(z)=\sum_{n=1}^{\infty} \alpha_{n} A_{n} \cos \left(\alpha_{n} z\right)
$$

The above function can be exhibited as a Fourier cosine series;

$$
\varphi^{\prime}(z)=\frac{f_{0}}{2}+\sum_{n=1}^{\infty} f_{n} \cos \left(\alpha_{n} z\right)
$$

In the equation $(8), f_{0}$ and $f_{n}$ coefficients are indicated as follows.

$$
\begin{gathered}
f_{0}=\frac{2}{L} \int_{0}^{L} \varphi^{\prime}(z) \partial z=\frac{2}{L}(\varphi(L)-\varphi(0)) \\
f_{n}=\frac{2}{L} \int_{0}^{L} \varphi^{\prime}(z) \cos \left(\alpha_{n} z\right) \partial z \quad n=1,2,3 \ldots
\end{gathered}
$$

Finally, if partial integration is applied;

$$
\begin{gathered}
f_{n}=\frac{2}{L}\left[\varphi(z) \cos \left(\alpha_{n} z\right)\right]_{0}^{L}+\frac{2}{L}\left[\alpha_{n} \int_{0}^{L} \varphi(z) \sin \left(\alpha_{n} z\right) \partial z\right] \\
\left.f_{n}=\frac{2}{L}\left[(1)^{n} \varphi(L)\right)-\varphi(0)\right]+A_{n} \alpha_{n}
\end{gathered}
$$

The steps followed above are recognized as Stokes ' transformations. Higher order derivatives can be found out similarly. Up until the fourth order, resulting derivatives of the displacement function are obtained to be as following. 


$$
\begin{gathered}
\frac{\partial \varphi(z)}{\partial z}=\frac{\left(\varphi_{L}-\varphi_{0}\right)}{L}+\sum_{n=1}^{\infty} \cos \left(\alpha_{n} z\right)\left(\frac{2\left((-1)^{n} \varphi_{L}-\varphi_{0}\right)}{L}+\alpha_{n} A_{n}\right) \\
\frac{\partial^{2} \varphi(z)}{\partial z^{2}}=-\sum_{n=1}^{\infty} \alpha_{n} \sin \left(\alpha_{n} z\right)\left(\frac{2\left((-1)^{n} \varphi_{L}-\varphi_{0}\right)}{L}+\alpha_{n} A_{n}\right) \\
\frac{\partial^{3} \varphi(z)}{\partial z^{3}}=\frac{\left(\varphi_{L}^{n}-\varphi_{0}^{n}\right)}{L}+\sum_{n=1}^{\infty} \cos \left(\alpha_{n} z\right)\left[\frac{2}{L}\left(\varphi_{L}^{n}(-1)^{n}-\varphi_{0}^{n}\right)-\alpha_{n}{ }^{2}\left(\frac{2}{L}\left(\varphi_{L}(-1)^{n}-\varphi_{0}\right)\right.\right. \\
\left.\left.+\alpha_{n} A_{n}\right)\right] \\
\frac{\partial^{4} \varphi(z)}{\partial z^{4}}=-\sum_{n=1}^{\infty} \sin \left(\alpha_{n} z\right)\left[\frac{2}{L}\left(\varphi_{L}{ }^{\prime \prime}(-1)^{n}-\varphi_{0}{ }^{n}\right)-\alpha_{n}{ }^{2}\left(\frac{2}{L}\left(\varphi_{L}(-1)^{n}-\varphi_{0}\right)+\alpha_{n} A_{n}\right)\right] \alpha_{n}
\end{gathered}
$$

The first derivative of the rotation function yields;

$$
\theta^{\prime}(z)=-\sum_{n=1}^{\infty} \alpha_{n} B_{n} \sin \left(\alpha_{n} z\right)
$$

In order to display the above function as a Fourier cosine series, the second derivative of the rotation function is necessary to be calculated;

$$
\theta^{n}(z)=-\sum_{n=1}^{\infty} \alpha_{n}^{2} B_{n} \cos \left(\alpha_{n} z\right)
$$

If the steps followed for the displacement function are similarly applied for the rotation function up until the third order, resulting derivatives of the rotation function are obtained to be as following.

$$
\begin{gathered}
\frac{\partial \theta(z)}{\partial z}=-\sum_{n=1}^{\infty} \alpha_{n} B_{n} \sin \left(\alpha_{n} z\right) \\
\frac{\partial^{2} \theta(z)}{\partial z^{2}}=\frac{\left(\theta_{L}^{\prime}-\theta_{0}^{\prime}\right)}{L}+\sum_{n=1}^{\infty} \cos \left(\alpha_{n} z\right)\left(\frac{2\left((-1)^{n} \theta_{L}^{\prime}-\theta_{0}^{\prime}\right)}{L}-\alpha_{n}{ }^{2} B_{n}\right) \\
\frac{\partial^{3} \theta(z)}{\partial z^{3}}=\sum_{n=1}^{\infty} \alpha_{n} \sin \left(\alpha_{n} z\right)\left(\frac{2\left(\theta_{0}{ }^{\prime}-\theta_{L}{ }^{\prime}(-1)^{n}\right)}{L}+\alpha_{n}{ }^{2} B_{n}\right)
\end{gathered}
$$

\subsection{Fourier Coefficients}

Taking the first derivative of the equation (1);

$$
E I \frac{\partial^{3} \theta}{\partial z^{3}}+\gamma^{2} P \frac{\partial^{4} \varphi}{\partial z^{4}}-\kappa_{S} G A\left(\frac{\partial \theta}{\partial z}+\frac{\partial^{2} \varphi}{\partial z^{2}}\right)=0
$$


If the equations (14), (16), (19) and (21) are written in the equations (2) and (22);

$$
\begin{aligned}
& \begin{array}{c}
E I\left(\frac{2\left(\theta_{0}{ }^{\prime}-\theta_{L}{ }^{\prime}(-1)^{n}\right)}{L}+\alpha_{n}{ }^{2} B_{n}\right) \alpha_{n} \sin \left(\alpha_{n} z\right)-\gamma^{2} P\left[\frac{2}{L}\left(\varphi_{L}^{\prime \prime}(-1)^{n}-\varphi_{0}^{n \prime}\right)\right. \\
\left.-\alpha_{n}{ }^{2}\left(\frac{2}{L}\left(\varphi_{L}(-1)^{n}-\varphi_{0}\right)+\alpha_{n} A_{n}\right)\right] \alpha_{n} \sin \left(\alpha_{n} z\right)-\kappa_{S} G A\left(-\alpha_{n} B_{n} \sin \left(\alpha_{n} z\right)\right. \\
\left.\quad-\alpha_{n} \sin \left(\alpha_{n} z\right)\left(\frac{2\left((-1)^{n} \varphi_{L}-\varphi_{0}\right)}{L}+\alpha_{n} A_{n}\right)\right)=0 \\
\kappa_{S} G A\left(-\alpha_{n} B_{n} \sin \left(\alpha_{n} z\right)-\alpha_{n} \sin \left(\alpha_{n} z\right)\left(\frac{2\left((-1)^{n} \varphi_{L}-\varphi_{0}\right)}{L}+\alpha_{n} A_{n}\right)\right) \\
\quad+P \alpha_{n} \sin \left(\alpha_{n} z\right)\left(\frac{2\left((-1)^{n} \varphi_{L}-\varphi_{0}\right)}{L}+\alpha_{n} A_{n}\right)=0
\end{array}
\end{aligned}
$$

$A_{n}$ and $B_{n}$ are derived from these equations when $\varphi_{0}$ and $\varphi_{L}$ are equal to zero.

$$
\begin{gathered}
A_{n}=-\frac{2 A G \kappa_{S}\left(P \gamma^{2} \varphi_{0}{ }^{\prime \prime}+E I \theta_{0}{ }^{\prime}+(-1)^{n+1}\left(P \gamma^{2} \varphi_{L}{ }^{\prime \prime}+E I \theta_{L}{ }^{\prime}\right)\right)}{E I L P \alpha_{n}{ }^{3}+A G L \kappa_{s} \alpha_{n}\left(P+\left(-E I+P \gamma^{2}\right) \alpha_{n}{ }^{2}\right)} \\
B_{n}=-\frac{2\left(P-A G \kappa_{S}\right)\left(P \gamma^{2} \varphi_{0}{ }^{\prime \prime}+E I \theta_{0}{ }^{\prime}+(-1)^{n+1}\left(P \gamma^{2} \varphi_{L}{ }^{\prime \prime}+E I \theta_{L}{ }^{\prime}\right)\right)}{E I L P \alpha_{n}{ }^{2}+A G L \kappa_{S}\left(P+\left(-E I+P \gamma^{2}\right) \alpha_{n}{ }^{2}\right)}
\end{gathered}
$$

\subsection{Boundary Conditions}

Flexural moment function $\mathrm{M}(\mathrm{x})$ of nonlocal Timoshenko beams is shown as following [7].

$$
M=E I \frac{\partial \theta}{\partial z}+P \gamma^{2} \frac{\partial^{2} \varphi}{\partial z^{2}}
$$

If the equation (27) is written in the equations (25) and (26);

$$
\begin{gathered}
A_{n}=-\frac{2 A G \kappa_{S}\left(M_{0}+(-1)^{n+1}\left(M_{L}\right)\right)}{E I L P \alpha_{n}{ }^{3}+A G L \kappa_{S} \alpha_{n}\left(P+\left(-E I+P \gamma^{2}\right) \alpha_{n}{ }^{2}\right)} \\
B_{n}=-\frac{2\left(P-A G \kappa_{S}\right)\left(M_{0}+(-1)^{n+1}\left(M_{L}\right)\right)}{E I L P \alpha_{n}{ }^{2}+A G L \kappa_{S}\left(P+\left(-E I+P \gamma^{2}\right) \alpha_{n}{ }^{2}\right)}
\end{gathered}
$$

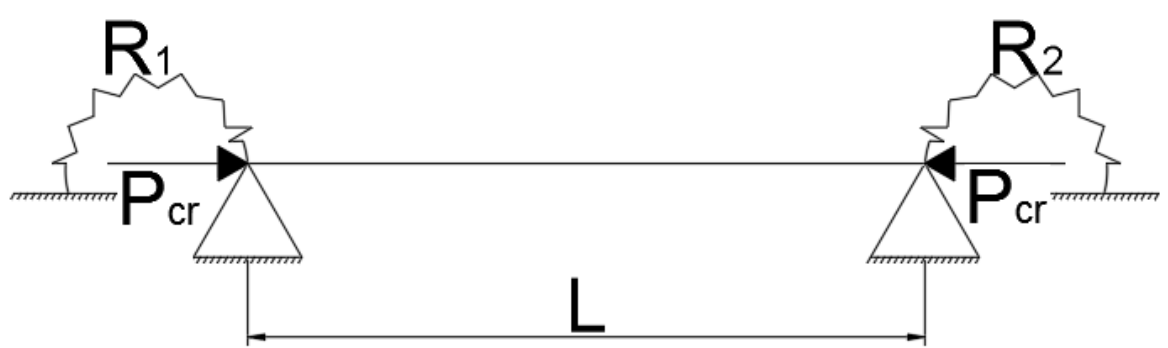

Fig. 1. The figure, displaying boundary conditions. 
Flexural moment function $\mathrm{M}(\mathrm{x})$ can be shown in the form;

$$
M=\theta R \rightarrow M-\theta R=0,
$$

where $\mathrm{R}$ is stiffness of the rotational springs.

\subsection{Construction of Coefficients Matrix}

If $\mathrm{z}=0$ and $\alpha_{\mathrm{n}}=\mathrm{n} \pi / \mathrm{L}$ are written in the equation (30);

$$
\left.0=M_{0}+\sum_{n=1}^{\infty} R_{1}\left(\frac{2\left(P-A G \kappa_{S}\right)\left(M_{0}+(-1)^{n+1}\left(M_{L}\right)\right)}{\operatorname{EILP}\left(\frac{n \pi}{L}\right)^{2}+A G L \kappa_{S}\left(P+\left(-E I+P \gamma^{2}\right)\left(\frac{n \pi}{L}\right)^{2}\right)} \cos \frac{n \pi}{L} z\right)\right)
$$

If $\mathrm{z}=\mathrm{L}$ and $\alpha_{\mathrm{n}}=\mathrm{n} \pi / \mathrm{L}$ are written in the equation (30);

$$
\left.0=M_{L}+\sum_{\mathrm{n}=1}^{\infty} R_{2}\left(\frac{2\left(P-A G \kappa_{S}\right)\left(M_{0}+(-1)^{n+1}\left(M_{L}\right)\right)}{\operatorname{EILP}\left(\frac{\mathrm{n} \pi}{\mathrm{L}}\right)^{2}+A G L \kappa_{S}\left(P+\left(-E I+P \gamma^{2}\right)\left(\frac{\mathrm{n} \pi}{\mathrm{L}}\right)^{2}\right)} \cos \frac{\mathrm{n} \pi}{\mathrm{L}} \mathrm{z}\right)\right)
$$

Two equations are obtained dependent on $\mathrm{M}_{0}$ and $\mathrm{M}_{\mathrm{L}}$. Thus coefficients matrix is composed.

$$
\left[\begin{array}{ll}
\Phi_{11} & \Phi_{12} \\
\Phi_{21} & \Phi_{22}
\end{array}\right]\left[\begin{array}{l}
M_{0} \\
M_{L}
\end{array}\right]=0
$$

Where $\Phi_{11}, \Phi_{12}, \Phi_{21}$ and $\Phi_{22}$ parameters are given the below.

$$
\begin{gathered}
\Phi_{11}=-1-\sum_{\mathrm{n}=1}^{\infty} \frac{2 L R_{1}\left(P-A G \kappa_{S}\right)}{E I P \pi^{2} \mathrm{n}^{2}+A G\left(P L^{2}+\pi^{2} \mathrm{n}^{2}\left(-E I+P \gamma^{2}\right)\right) \kappa_{S}} \\
\Phi_{12}=\sum_{\mathrm{n}=1}^{\infty} \frac{2(-1)^{n} L R_{1}\left(P-A G \kappa_{S}\right)}{E I P \pi^{2} \mathrm{n}^{2}+A G\left(P L^{2}+\pi^{2} \mathrm{n}^{2}\left(-E I+P \gamma^{2}\right)\right) \kappa_{S}} \\
\Phi_{21}=-1-\sum_{\mathrm{n}=1}^{\infty} \frac{2 L R_{2}\left(P-A G \kappa_{S}\right)}{E I P \pi^{2} \mathrm{n}^{2}+A G\left(P L^{2}+\pi^{2} \mathrm{n}^{2}\left(-E I+P \gamma^{2}\right)\right) \kappa_{S}} \\
\Phi_{22}=\sum_{\mathrm{n}=1}^{\infty} \frac{2(-1)^{n} L R_{2}\left(P-A G \kappa_{S}\right)}{E I P \pi^{2} \mathrm{n}^{2}+A G\left(P L^{2}+\pi^{2} \mathrm{n}^{2}\left(-E I+P \gamma^{2}\right)\right) \kappa_{S}}
\end{gathered}
$$




\section{Numerical results}

The equation (33) is an eigenvalue problem. The critical buckling loads can be calculated by setting the determinant of the coefficient matrix to zero. The characteristic equation can be achieved by assigning the proper values of $\left(R_{1}\right)$ and $\left(R_{2}\right)$ corresponding to the restrained boundary condition. If $R_{1}=0$ and $R_{2}=0$, the beam is pinned ended beam. If $R_{1}=0$ and $R_{2}=\infty$, the beam is clamped pinned beam. If $R_{1}=\infty$ and $R_{2}=\infty$, the beam is clamped ended beam. $\kappa_{s}=0.9$ for Timoshenko beam, $\kappa_{s}=\infty$ for Euler Bernoulli beam.

With the theoretical formulation proposed in this study, different numerical examples are solved in this part. Firstly, accuracy and validity of the present mathematical approach is validated. Validation study is given in Tables 1-2.

Several numerical examples are solved numerically. Tabulated values given in the Table 1 are plotted in the Figs 2-4. The Fourier sine and cosine series are truncated for 50 terms of infinite series. It can be seen from these figures, the nonlocal effects are more pronounced for higher buckling modes.

Table 1. Critical buckling loads $\operatorname{Pcr}(\mathrm{nN})$ for pinned, clamped-pinned and clamped beams with diverse small scale coefficients $\gamma(\mathrm{nm})$ and length-to-diameter ratios $\mathrm{L} / \mathrm{d}$ in this study.

\begin{tabular}{|c|c|c|c|c|c|c|c|c|c|c|}
\hline \multirow{2}{*}{$\begin{array}{c}\gamma(\mathrm{nm}) \\
\mathrm{L} / \mathrm{d}\end{array}$} & \multicolumn{2}{|c|}{0} & \multicolumn{2}{|c|}{0.5} & \multicolumn{2}{|c|}{1} & \multicolumn{2}{|c|}{1.5} & \multicolumn{2}{|c|}{2} \\
\hline & $\mathrm{T}$ & EB & $\mathrm{T}$ & EB & $\mathrm{T}$ & EB & $\mathrm{T}$ & EB & $\mathrm{T}$ & EB \\
\hline \multicolumn{11}{|c|}{ Pinned ended beam } \\
\hline 10 & 4.7670 & 4.8447 & 4.6540 & 4.7281 & 4.3450 & 4.4095 & 3.9121 & 3.9644 & 3.4333 & 3.4735 \\
\hline 12 & 3.3267 & 3.3644 & 3.2713 & 3.3077 & 3.1156 & 3.1486 & 2.8865 & 2.9149 & 2.6172 & 2.6405 \\
\hline 14 & 2.4514 & 2.4718 & 2.4212 & 2.4411 & 2.3348 & 2.3533 & 2.2038 & 2.2202 & 2.0432 & 2.0574 \\
\hline 16 & 1.8805 & 1.8925 & 1.8626 & 1.8744 & 1.8111 & 1.8222 & 1.7313 & 1.7414 & 1.6306 & 1.6396 \\
\hline 18 & 1.4878 & 1.4953 & 1.4766 & 1.4840 & 1.4440 & 1.4511 & 1.3928 & 1.3993 & 1.3269 & 1.3329 \\
\hline \multicolumn{11}{|c|}{ Clamped-pinned beam } \\
\hline 10 & 9.6851 & 10.01 & 9.2298 & 9.5258 & 8.0890 & 8.3155 & 6.7074 & 6.8624 & 5.4130 & 5.5135 \\
\hline 12 & 6.7934 & 6.9525 & 6.5662 & 6.7147 & 5.9675 & 6.0899 & 5.1803 & 5.2723 & 4.3727 & 4.4381 \\
\hline 14 & 5.0215 & 5.1079 & 4.8963 & 4.9784 & 4.5555 & 4.6685 & 4.0820 & 4.1388 & 3.5643 & 3.6067 \\
\hline 16 & 3.8599 & 3.9107 & 3.7855 & 3.8343 & 3.5785 & 3.6221 & 3.2796 & 3.3163 & 2.9363 & 2.9656 \\
\hline 18 & 3.0581 & 3.0899 & 3.0112 & 3.0421 & 2.8788 & 2.9069 & 2.6821 & 2.7066 & 2.4481 & 2.4684 \\
\hline \multicolumn{11}{|c|}{ Clamped ended beam } \\
\hline 10 & 18.542 & 19.777 & 16.942 & 17.967 & 13.458 & 14.097 & 10.023 & 10.373 & 7.3846 & 7.5729 \\
\hline 12 & 13.127 & 13.734 & 12.304 & 12.836 & 10.357 & 10.731 & 8.1958 & 8.4284 & 6.3426 & 6.4810 \\
\hline 14 & 9.7591 & 10.090 & 9.2970 & 9.5974 & 8.1406 & 8.3700 & 6.7428 & 6.8995 & 5.4361 & 5.5374 \\
\hline 16 & 7.5298 & 7.7256 & 7.2517 & 7.4332 & 6.5283 & 6.6751 & 5.5977 & 5.7053 & 4.6665 & 4.7410 \\
\hline 18 & 5.9813 & 6.1042 & 5.8045 & 5.9202 & 5.3316 & 5.4291 & 4.6943 & 4.7696 & 4.0213 & 4.0765 \\
\hline
\end{tabular}


Table 2. In Ref. [7], critical buckling loads $\operatorname{Pcr}(\mathrm{nN})$ for pinned, clamped-pinned and clamped beams with diverse small scale coefficients $\gamma(\mathrm{nm})$ and length-to-diameter ratios $\mathrm{L} / \mathrm{d}$. $\mathrm{d}=1 \mathrm{~nm}, \mathrm{~A}=\pi^{2} / 4 \mathrm{~nm}^{2}, \mathrm{I}=\pi / 64 \mathrm{~nm}^{4}, \mathrm{E}=1000 \mathrm{kPa}, \mathrm{G}=420.168 \mathrm{kPa}$

\begin{tabular}{|c|c|c|c|c|c|c|c|c|c|c|}
\hline \multirow{2}{*}{$\begin{array}{c}\gamma \\
\mathrm{L} / \mathrm{d}\end{array}$} & \multicolumn{2}{|c|}{0} & \multicolumn{2}{|c|}{0.5} & \multicolumn{2}{|c|}{1} & \multicolumn{2}{|c|}{1.5} & \multicolumn{2}{|c|}{2} \\
\hline & $\mathrm{T}$ & EB & $\mathrm{T}$ & EB & $\mathrm{T}$ & EB & $\mathrm{T}$ & EB & $\mathrm{T}$ & EB \\
\hline \multicolumn{11}{|c|}{ Pinned ended beam } \\
\hline 10 & 4.7670 & 4.8447 & 4.6540 & 4.7281 & 4.3450 & 4.4095 & 3.9121 & 3.9644 & 3.4333 & 3.4735 \\
\hline 12 & 3.3267 & 3.3644 & 3.2713 & 3.3077 & 3.1156 & 3.1486 & 2.8865 & 2.9149 & 2.6172 & 2.6405 \\
\hline 14 & 2.4514 & 2.4718 & 2.4212 & 2.4411 & 2.3348 & 2.3533 & 2.2038 & 2.2202 & 2.0432 & 2.0574 \\
\hline 16 & 1.8805 & 1.8925 & 1.8626 & 1.8744 & 1.8111 & 1.8222 & 1.7313 & 1.7414 & 1.6306 & 1.6396 \\
\hline 18 & 1.4878 & 1.4953 & 1.4766 & 1.4840 & 1.4440 & 1.4511 & 1.3928 & 1.3993 & 1.3269 & 1.3329 \\
\hline \multicolumn{11}{|c|}{ Clamped-pinned beam } \\
\hline 10 & 9.5605 & 9.9155 & 9.1179 & 9.4349 & 8.0055 & 8.2461 & 6.6520 & 6.8151 & 5.3782 & 5.4830 \\
\hline 12 & 6.7118 & 6.8858 & 6.4904 & 6.6496 & 5.9059 & 6.0363 & 5.1348 & 5.2321 & 4.3410 & 4.4096 \\
\hline 14 & 4.9638 & 5.0589 & 4.8416 & 4.9297 & 4.5086 & 4.5844 & 4.0448 & 4.1052 & 3.5355 & 3.5811 \\
\hline 16 & 3.8168 & 3.8715 & 3.7441 & 3.7967 & 3.5418 & 3.5885 & 3.2490 & 3.2880 & 2.9120 & 2.9431 \\
\hline 18 & 3.0248 & 3.0603 & 2.9789 & 3.0121 & 2.8493 & 2.8795 & 2.6567 & 2.6828 & 2.4270 & 2.4489 \\
\hline \multicolumn{11}{|c|}{ Clamped ended beam } \\
\hline 10 & 18.192 & 19.379 & 16.649 & 17.638 & 13.273 & 13.894 & 9.9200 & 10.263 & 7.3283 & 7.5137 \\
\hline 12 & 12.874 & 13.458 & 12.082 & 12.594 & 10.199 & 10.562 & 8.0964 & 8.3233 & 6.2829 & 6.4187 \\
\hline 14 & 9.5687 & 9.8872 & 9.1240 & 9.4132 & 8.0077 & 8.2296 & 6.6514 & 6.8038 & 5.3765 & 5.4756 \\
\hline 16 & 7.3818 & 7.5699 & 7.1143 & 7.2889 & 6.4168 & 6.5585 & 5.5155 & 5.6199 & 4.6092 & 4.6819 \\
\hline 18 & 5.8631 & 5.9811 & 5.6931 & 5.8043 & 5.2375 & 5.3315 & 4.6212 & 4.6942 & 3.9675 & 4.0212 \\
\hline
\end{tabular}

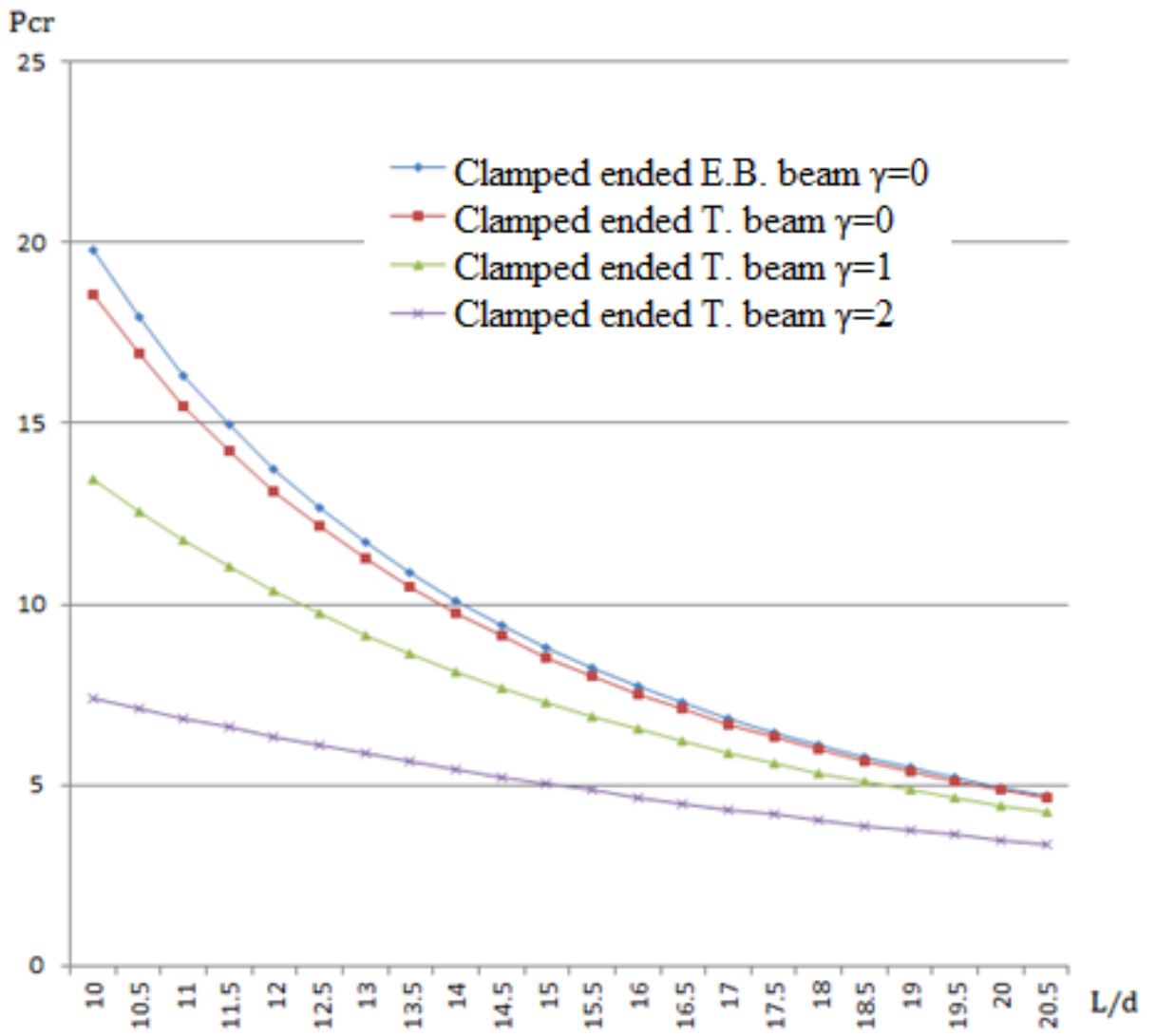

Fig. 2. Variations of critical buckling load for clamped ended beam. 


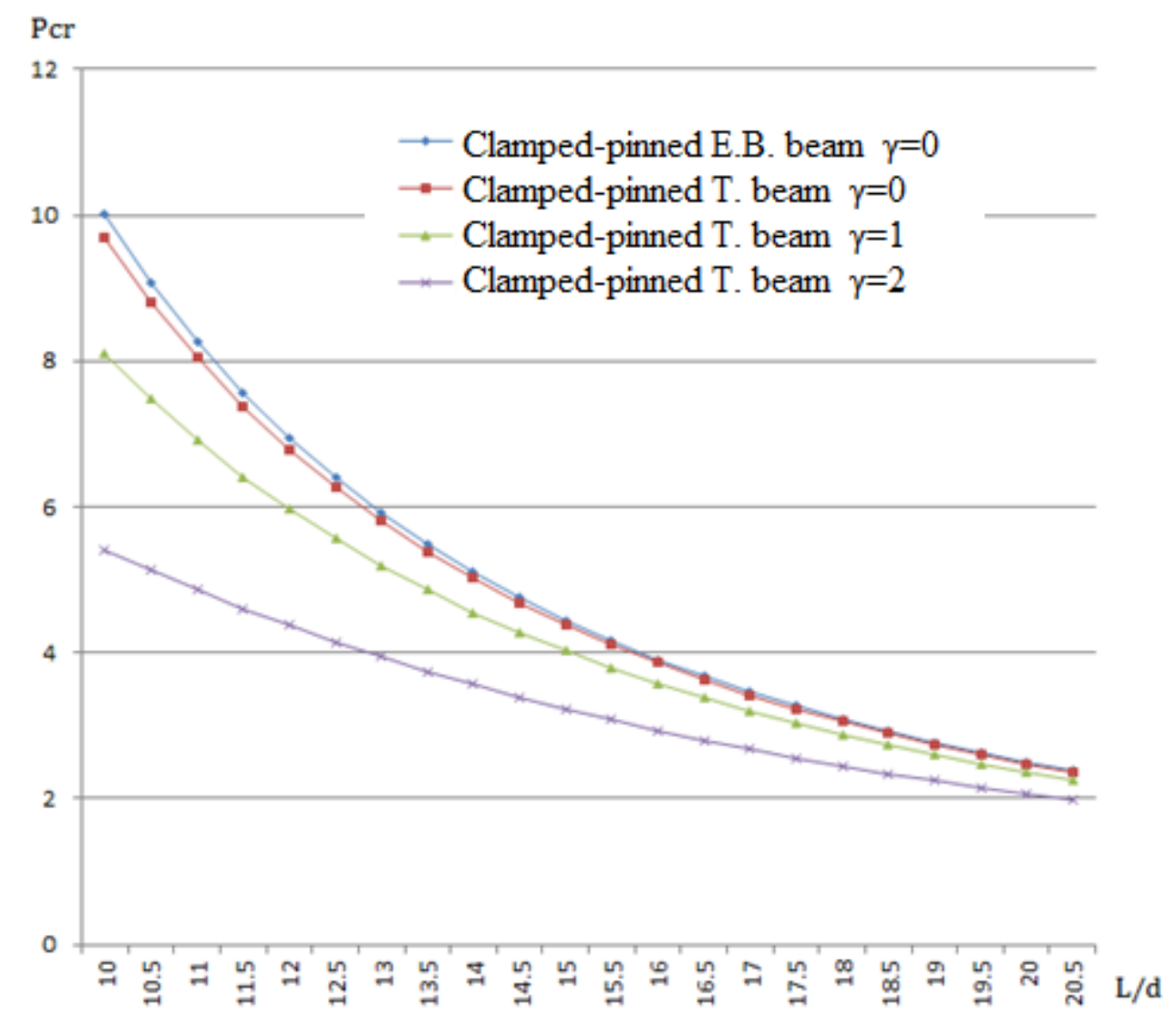

Fig. 3. Variations of critical buckling load for clamped pinned beam.

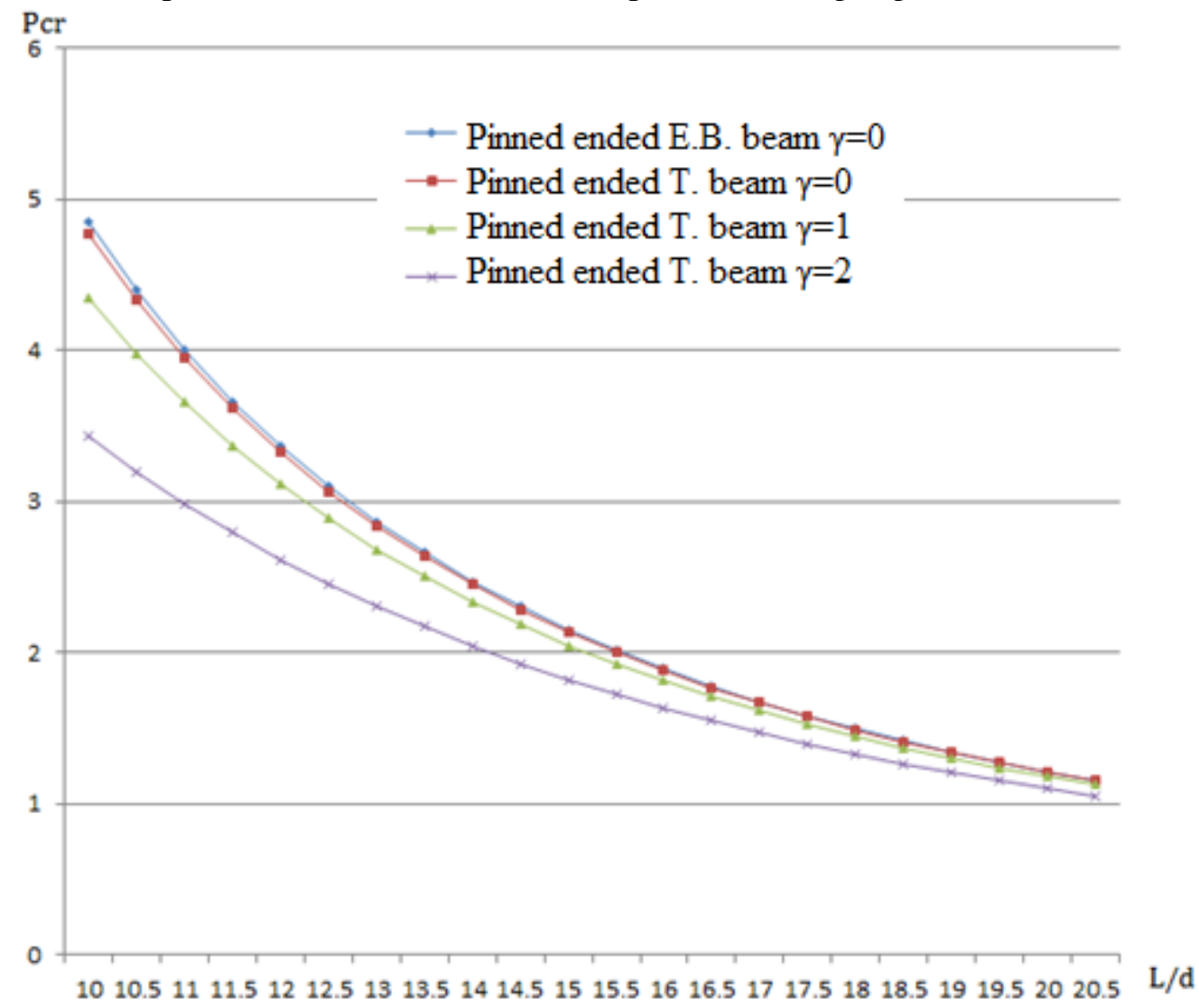

Fig. 4. Variations of critical buckling load for pinned ended beam. 


\section{Conclusions}

The critical buckling loads are found by using nonlocal elasticity theory for the Timoshenko and Euler Bernoulli beams. If the critical buckling loads of the Timoshenko and Euler Bernoulli beams are compared between each other, it is observed that. Once length-to-diameter ratios decline, results diverge from each other. Once length-to-diameter ratios increase, results converge to each other. Even if the results converge to each other. The critical buckling loads of the Timoshenko beams are always lower than the Euler Bernoulli beams. Because, in the Timoshenko beams, influence of shear on bending deformation is taken into account. Moreover, if the value of the small effect scale rises, the critical buckling loads decrease.

The results are compared to other studies found in the literature which led to the conclusion that a great deal of similarity exists between them, which additionally proves the accuracy of this method. Moreover, this method allows calculation with non-rigid boundary conditions. It is this reason that makes this study a significant contribution with a potential to pave the way for further and more advanced studies on this topic.

\section{References}

[1] Eringen, A. C., Nonlocal polar elastic continua, International journal of engineering science. 10, $1-16,1972$.

[2] Eringen A. C. and Edelen D. G. B., On nonlocal elasticity. Int. J. Eng. Sci, 10, 233-48, 1972.

[3] Eringen A. C., On differential equations of nonlocal elasticity and solutions of screw dislocation and surface waves, J. Appl. Phys. 54, 4703-4710, 1983.

[4] Li, C. Y., Chou T. W., A structural mechanics approach for the analysis of carbon nanotubes, Int. J. Solids Struct. 40, 2487-2499, 2003.

[5] Chowdhury, R., Adhikari, S., Wang, C. W., Scarpa, F., A molecular mechanics approach for the vibration of single walled carbon nanotubes. Comput. Mater. Sci., 48, 730-735, 2010.

[6] Poncharal, P., Wang, Z. L., Ugarte, D., Heer, W. A. D., Electrostatic deflections and electromechanical resonances of carbon nanotubes. Science, 283, 1513-1516, 1999.

[7] Wang, C. M., Zhang, Y. Y., Ramesh, S. S., Kitipornchai, S., Buckling analysis of micro-and nano-rods/tubes based on nonlocal Timoshenko beam theory. Journal of Physics D: Applied Physics, 17, 3904-3909, 2006.

[8] Ghannadpour, S. A. M., and Mohammadi, B., Buckling analysis of micro-and nano-rods/tubes based on nonlocal Timoshenko beam theory using Chebyshev polynomials. Advanced Materials Research, 123, 619-622, 2010.

[9] Yayli M. Ö., Buckling Analysis of a Rotationally Restrained Single Walled Carbon Nanotube Embedded In An Elastic Medium Using Nonlocal Elasticity. Int J Eng Appl Sci, 8(2), 40-50, 2016. [10] Yayli M. Ö., An Analytical Solution for Free Vibrations of A Cantilever Nanobeam with A Spring Mass System. Int J Eng Appl Sci, 7(4), 10-18, 2016.

[11] Civalek, Ö., Akgöz, B., Free vibration analysis of microtubules as cytoskeleton components: nonlocal Euler-Bernoulli beam modeling. Sci. Iranica Trans. B: Mech. Eng., 17, 367-375, 2010.

[12] Civalek, Ö., Demir, Ç., Bending analysis of microtubules using nonlocal Euler-Bernoulli beam theory. Appl. Math. Model., 35, 2053-2067, 2011.

[13] Wang, C.M., Kitipornchai, S., Lim, C.W., Eisenberger, M., Beam bending solutions based on nonlocal Timoshenko beam theory. J. Eng. Mech., 134, 475-481, 2008.

[14] Lu, P., Lee, H.P., Lu, C., Zhang, P.Q., Dynamic properties of flexural beams using a nonlocal elasticity model. J. Appl. Phys., 99, 73510-73518, 2006. 
[15] Murmu, T., Pradhan, S.C., Small-scale effect on the vibration of nonuniform nanocantilever based on nonlocal elasticity theory. Physica E, 41, 1451-1456, 2009.

[16] Rahmani, O., Pedram, O., Analysis and modeling the size effect on vibration of functionally graded nanobeams based on nonlocal Timoshenko beam theory. Int. J. Eng. Sci, 77, 55-70, 2014.

[17] Eltaher, M.A., Emam, S.A., Mahmoud, F.F., Static and stability analysis of nonlocal functionally graded nanobeams. Compos. Struct, 96, 82-88, 2013

[18] Thai, H.T., A nonlocal beam theory for bending, buckling, and vibration of nanobeams. Int. J. Eng. Sci., 52, 56-64, 2012.

[19] Reddy J. N., Pang, S. D., Nonlocal continuum theories of beam for the analysis of carbon nanotubes. Journal of Applied Physics, 103, 1-16, 2008.

[20] Setoodeh, A.R., Khosrownejad, M., Malekzadeh, P., Exact nonlocal solution for post buckling of single-walled carbon nanotubes. Physica E, 43, 1730-1737, 2011

[21] Yayli, M.Ö., Buckling Analysis of a Rotationally Restrained Single Walled Carbon Nanotube. Acta Physica Polonica A, 127, 3, 678-683, 2015.

[22] Yayli, M.Ö., Stability analysis of gradient elastic microbeams with arbitrary boundary conditions. Journal of Mechanical Science and Technology, 29, 8, 3373-3380, 2015.

[23] Artan R., Tepe A., The initial values method for buckling of nonlocal bars with application in nanotechnology. European Journal of Mechanics-A/Solids, 27, (3), 469-477, 2008.

[24] Demir, Ç., Civalek, Ö., Nonlocal finite element formulation for vibration. International Journal of Engineering and Applied Sciences, 8(2), 109-117, 2016.

[25] Demir, Ç., Civalek, Ö., On the analysis of microbeams. International Journal of Engineering Science, 121(Supplement C), 14-33, 2017.

[26] Demir, Ç., Civalek, Ö., Nonlocal deflection of microtubules under point load. International Journal of Engineering and Applied Sciences, 7(3), 33-39, 2015

[27] Akgoz, B., Civalek, Ö., Buckling analysis of linearly tapered micro-columns based on strain gradient elasticity. Structural Engineering and Mechanics, 48(2), 195-205, 2013.

[28] Akgöz, B., Civalek, Ö., Bending analysis of embedded carbon nanotubes resting on an elastic foundation using strain gradient theory. Acta Astronautica 119, 1-12, 2016.

[29] Civalek, Ö., Demir Ç., A simple mathematical model of microtubules surrounded by an elastic matrix by nonlocal finite element method. Applied Mathematics and Computation 289, 335$352,2016$.

[30] Mercan, K., Civalek, Ö., DSC method for buckling analysis of boron nitride nanotube (BNNT) surrounded by an elastic matrix. Composite Structures 143, 300-309, 2016.

[31] Civalek, Ö., Analysis of thick rectangular plates with symmetric cross-ply laminates based on first-order shear deformation theory. Journal of Composite Materials 42(26), 2853-2867, 2008.

[32] Yayli, M.Ö., On the axial vibration of carbon nanotubes with different boundary conditions. Micro \& Nano Letters 9(11), 807-811, 2014.

[33] Yayli, M.Ö., Torsion of nonlocal bars with equilateral triangle cross sections. Journal of Computational and Theoretical Nanoscience 10(2), 376-379, 2013.

[34] Yayli, M.Ö., Weak formulation of finite element method for nonlocal beams using additional boundary conditions. Journal of Computational and Theoretical Nanoscience 8(11), 2173-2180, 2011.

[35] Yayli, M.Ö., Çerçevik A. E., 1725. Axial vibration analysis of cracked nanorods with arbitrary boundary conditions. Journal of Vibroengineering 17(6), 2907-2921, 2015.

[36] Yayli, M.Ö., Airy Stress Functions for Transverse Sinusoidally Loaded Beam in Nonlocal Elasticity. Journal of Computational and Theoretical Nanoscience 8(10), 2006-2012, 2011. 